Stud. Univ. Babeş-Bolyai Math. 66(2021), No. 1, 17-27

DOI: 10.24193/subbmath.2021.1.02

\title{
On some evolution inclusions in non separable Banach spaces
}

\author{
Aurelian Cernea
}

Dedicated to Professor Gheorghe Moroşanu on the occasion of his 70th anniversary.

\begin{abstract}
We study a Cauchy problem of a class of nonconvex second-order integro-differential inclusions and a boundary value problem associated to a semilinear evolution inclusion defined by nonlocal conditions in non-separable Banach spaces. The existence of mild solutions is established under Filippov type assumptions.
\end{abstract}

Mathematics Subject Classification (2010): 34A60.

Keywords: Lusin measurable multifunctions, selection, mild solution.

\section{Introduction}

In this note we study two classes of evolution differential inclusions. First we consider the problem

$$
x^{\prime \prime}(t) \in A(t) x(t)+\int_{0}^{t} K(t, s) F(s, x(s)) d s, \quad x(0)=x_{0}, x^{\prime}(0)=y_{0},
$$

where $F:[0, T] \times X \rightarrow \mathcal{P}(X)$ is a set-valued map lipschitzian with respect to the second variable, $X$ is a Banach space, $\{A(t)\}_{t \geq 0}$ is a family of linear closed operators from $X$ into $X$ that genearates an evolution system of operators $\{G(t, s)\}_{t, s \in[0, T]}$, $\Delta=\{(t, s) \in[0, T] \times[0, T] ; t \geq s\}, K(.,):. \Delta \rightarrow \mathbb{R}$ is continuous and $x_{0}, y_{0} \in X$. The general framework of evolution operators $\{A(t)\}_{t \geq 0}$ that define problem (1.1) has been developed by Kozak ([19]) and improved by Henriquez ([17]).

Existence results and some qualitative properties of the mild solutions of problem (1.1) may be found in [14] in the case when $X$ is a separable Banach space.

De Blasi and Pianigiani ([15]) obtained the existence of mild solutions for semilinear differential inclusions on an arbitrary, not necessarily separable, Banach space $X$. Even if Filippov's ideas ([16]) are still present, the approach in [15] is fundamental 
different: it consists in the construction of the measurable selections of the multifunction. This construction does not use classical selection theorems such as Kuratowski and Ryll-Nardzewski's ([20]) or Bressan and Colombo's ([7]).

The aim of this note is to obtain an existence result for problem (1.1) similar to the one in [15]. We will prove the existence of solutions for problem (1.1) in an arbitrary space $X$ under Filippov-type assumptions on $F$.

In several recent papers $([2,3,5,12,13,17,18])$ existence results and qualitative properties of mild solutions have been obtained for the following problem

$$
x^{\prime \prime}(t) \in A(t) x(t)+F(t, x(t)), \quad x(0)=x_{0}, x^{\prime}(0)=y_{0},
$$

with $A($.$) and F(.,$.$) as above.$

On one hand, the result in the present paper extends to the integro-differential framework (1.1) the result in [12] obtained for problem (1.2) and, on the other hand, this paper extends to second-order integro-differential inclusions a similar result in [10] obtained for a class of first-order integro-differential inclusions.

The second class of evolution inclusions that we are considering is

$$
\begin{gathered}
x^{\prime} \in A x+F(t, x) \quad \text { a.e. }([0, T]), \\
x(0)+\sum_{i=1}^{m} a_{i} x\left(t_{i}\right)=x_{0},
\end{gathered}
$$

where $X$ is a real separable Banach space, $a_{i} \in \mathbb{R}, a_{i} \neq 0, i=\overline{1, m}, x_{0} \in X$, $0<t_{1}<t_{2}<\ldots<t_{m}<T, F:[0, T] \times X \rightarrow \mathcal{P}(X)$ is a set-valued map and $A$ is the infinitesimal generator of a linear semigroup $\{\mathcal{G}(t) ; t \geq 0\}$.

The nonlocal condition (1.4) was used by Byszewski ([8, 9]). If $a_{i} \neq 0, i=\overline{1, m}$ the results can be applied in kinematics to determine the evolution $t \rightarrow x(t)$ of the location of a physical object for which the positions $x(0), x\left(t_{1}\right), \ldots, x\left(t_{m}\right)$ are unknown but it is known the condition (1.4). Consequently, to describe some physical phenomena the nonlocal condition may be more useful than the standard initial condition $x(0)=x_{0}$. Obviously, when $a_{i}=0, i=\overline{1, m}$, one has the classical initial condition.

Existence of mild solutions of problem (1.3)-(1.4) has been obtained in $[4,6]$ for convex as well as nonconvex set-valued maps. All these results are based on some suitable theorems of fixed point theory. In our recent paper [11] it is shown that Filippov's ideas $([1,16])$ can be suitably adapted in order to prove the existence of solutions to problem (1.3)-(1.4) provided the Banach space $X$ is separable.

The result that we established in non separable Banach spaces for problem (1.3)(1.4) may be interpreted as extension of the result in [15] from Cauchy problems to boundary value problems defined by nonlocal conditions and as an extension of the result in [11] to non separable Banach spaces.

The paper is organized as follows: in Section 2 we present the notations, definitions and preliminary results to be used in the sequel and in Section 3 we prove the main results. 


\section{Preliminaries}

Consider $X$, an arbitrary real Banach space with norm $|$.$| and with the corre-$ sponding metric $d(.,$.$) . Let \mathcal{P}(X)$ be the space of all bounded nonempty subsets of $X$ endowed with the Hausdorff pseudometric

$$
\mathrm{d}_{H}(A, B)=\max \left\{\mathrm{d}^{*}(A, B), \mathrm{d}^{*}(B, A)\right\}, \quad \mathrm{d}^{*}(A, B)=\sup _{a \in A} \mathrm{~d}(a, B),
$$

where $\mathrm{d}(x, A)=\inf _{a \in A}|x-a|, A \subset X, x \in X$.

Let $\mathcal{L}$ be the $\sigma$-algebra of the (Lebesgue) measurable subsets of $R$ and, for $A \in \mathcal{L}$, let $\mu(A)$ be the Lebesgue measure of $A$.

Let $X$ be a Banach space and $Y$ be a metric space. An open (resp., closed) ball in $Y$ with center $y$ and radius $r$ is denoted by $B_{Y}(y, r)$ (resp., $\bar{B}_{Y}(y, r)$. In what follows, $B=B_{X}(0,1)$.

A multifunction $F: Y \rightarrow \mathcal{P}(X)$ with closed bounded nonempty values is said to be $d_{H}$-continuous at $y_{0} \in Y$ if for every $\varepsilon>0$ there exists $\delta>0$ such that for any $y \in B_{Y}\left(y_{0}, r\right)$ there is $\mathrm{d}_{H}\left(F(y), F\left(y_{0}\right)\right) \leq \varepsilon . F$ is called $\mathrm{d}_{H}$-continuous if it is so at each point $y_{0} \in Y$.

Let $A \in \mathcal{L}$, with $\mu(A)<\infty$. A multifunction $F: Y \rightarrow \mathcal{P}(X)$ with closed bounded nonempty values is said to be Lusin measurable if for every $\varepsilon>0$ there exists a compact set $K_{\varepsilon} \subset A$, with $\mu\left(A \backslash K_{\varepsilon}\right)<\varepsilon$ such that $F$ restricted to $K_{\varepsilon}$ is $\mathrm{d}_{H}$-continuous.

It is clear that if $F, G: A \rightarrow \mathcal{P}(X)$ and $f: A \rightarrow X$ are Lusin measurable, then so are $F$ restricted to $B(B \subset A$ measurable), $F+G$ and $t \rightarrow \mathrm{d}(f(t), F(t))$. Moreover, the uniform limit of a sequence of Lusin measurable multifunctions is Lusin measurable, too.

Let $I$ stand for the interval $[0, T], T>0, C(I, X)$ is the Banach space of all continuous functions from $I$ to $X$ with the norm $\|x\|_{C}=\sup _{t \in I}|x(t)|$ and $L^{1}(I, X)$ is the Banach space of (Bochner) integrable functions $u():. I \rightarrow X$ endowed with the norm $\|u\|_{1}=\int_{0}^{T}|u(t)| d t$. Denote by $B(X)$ the Banach space of bounded linear operators from $X$ into $X$ with the norm $\|N\|=\sup \{|N(y)| ;|y|=1\}$.

In what follows $\{A(t)\}_{t \geq 0}$ is a family of linear closed operators from $X$ into $X$ that genearates an evolution system of operators $\{G(t, s)\}_{t, s \in I}$. By hypothesis the domain of $A(t), D(A(t))$ is dense in $X$ and is independent of $t$.

Definition 2.1. ([17, 19]) A family of bounded linear operators $G(t, s): X \rightarrow X$, $(t, s) \in \Delta:=\{(t, s) \in I \times I ; s \leq t\}$ is called an evolution operator of the equation

$$
x^{\prime \prime}(t)=A(t) x(t)
$$

if

i) For any $x \in X$, the map $(t, s) \rightarrow G(t, s) x$ is continuously differentiable and

a) $G(t, t)=0, t \in I$.

b) If $t \in I, x \in X$ then $\left.\frac{\partial}{\partial t} G(t, s) x\right|_{t=s}=x$ and $\left.\frac{\partial}{\partial s} G(t, s) x\right|_{t=s}=-x$.

ii) If $(t, s) \in \Delta$, then $\frac{\partial}{\partial s} G(t, s) x \in D(A(t))$, the map $(t, s) \rightarrow G(t, s) x$ is of class $C^{2}$ and

a) $\frac{\partial^{2}}{\partial t^{2}} G(t, s) x \equiv A(t) G(t, s) x$. 
b) $\frac{\partial^{2}}{\partial s^{2}} G(t, s) x \equiv G(t, s) A(t) x$.

c) $\left.\frac{\partial^{2}}{\partial s \partial t} G(t, s) x\right|_{t=s}=0$.

iii) If $(t, s) \in \Delta$, then there exist $\frac{\partial^{3}}{\partial t^{2} \partial s} G(t, s) x, \frac{\partial^{3}}{\partial s^{2} \partial t} G(t, s) x$ and tinuous.

a) $\frac{\partial^{3}}{\partial t^{2} \partial s} G(t, s) x \equiv A(t) \frac{\partial}{\partial s} G(t, s) x$ and the map $(t, s) \rightarrow A(t) \frac{\partial}{\partial s} G(t, s) x$ is con-

b) $\frac{\partial^{3}}{\partial s^{2} \partial t} G(t, s) x \equiv \frac{\partial}{\partial t} G(t, s) A(s) x$.

As an example for equation (2.1) one may consider the problem (e.g., [19])

$$
\begin{gathered}
\frac{\partial^{2} z}{\partial t^{2}}(t, \tau)=\frac{\partial^{2} z}{\partial \tau^{2}}(t, \tau)+a(t) \frac{\partial z}{\partial t}(t, \tau), \quad t \in[0, T], \tau \in[0,2 \pi], \\
z(t, 0)=z(t, \pi)=0, \quad \frac{\partial z}{\partial \tau}(t, 0)=\frac{\partial z}{\partial \tau}(t, 2 \pi), t \in[0, T],
\end{gathered}
$$

where $a():. I \rightarrow \mathbb{R}$ is a continuous function. This problem is modeled in the space $X=L^{2}(\mathbb{R}, \mathbb{C})$ of $2 \pi$-periodic 2-integrable functions from $\mathbb{R}$ to $\mathbb{C}, A_{1} z=\frac{d^{2} z(\tau)}{d \tau^{2}}$ with domain $H^{2}(\mathbb{R}, \mathbb{C})$ the Sobolev space of $2 \pi$-periodic functions whose derivatives belong to $L^{2}(\mathbb{R}, \mathbb{C})$. It is well known that $A_{1}$ is the infinitesimal generator of strongly continuous cosine functions $C(t)$ on $X$. Moreover, $A_{1}$ has discrete spectrum; namely the spectrum of $A_{1}$ consists of eigenvalues $-n^{2}, n \in \mathbb{Z}$ with associated eigenvectors

$$
z_{n}(\tau)=\frac{1}{\sqrt{2 \pi}} e^{i n \tau}, n \in \mathbb{N} .
$$

The set $z_{n}, n \in \mathbb{N}$ is an orthonormal basis of $X$. In particular,

$$
A_{1} z=\sum_{n \in \mathbb{Z}}-n^{2}<z, z_{n}>z_{n}, z \in D\left(A_{1}\right) .
$$

The cosine function is given by

$$
C(t) z=\sum_{n \in \mathbf{Z}} \cos (n t)<z, z_{n}>z_{n}
$$

with the associated sine function

$$
S(t) z=t<z, z_{0}>z_{0}+\sum_{n \in \mathbf{Z}^{*}} \frac{\sin (n t)}{n}<z, z_{n}>z_{n} .
$$

For $t \in I$ define the operator $A_{2}(t) z=a(t) \frac{d z(\tau)}{d \tau}$ with domain $D\left(A_{2}(t)\right)=H^{1}(\mathbb{R}, \mathbb{C})$. Set $A(t)=A_{1}+A_{2}(t)$. It has been proved in [19] that this family generates an evolution operator as in Definition 2.1.

Definition 2.2. A continuous mapping $x(.) \in C(I, X)$ is called a mild solution of problem (1.1) if there exists a (Bochner) integrable function $f(.) \in L^{1}(I, X)$ such that

$$
\begin{gathered}
f(t) \in F(t, x(t)) \text { a.e. }(I) \\
x(t)=-\frac{\partial}{\partial s} G(t, 0) x_{0}+G(t, 0) y_{0}+\int_{0}^{t} G(t, s) \int_{0}^{s} K(s, \tau) f(\tau) d \tau, t \in I .
\end{gathered}
$$


We shall call $(x(),. f()$.$) a trajectory-selection pair of (1.1)$ if $f($.$) verifies (2.2)$ and $x($.$) is defined by (2.3)$.

We note that condition (2.3) can be rewritten as

$$
x(t)=-\frac{\partial}{\partial s} G(t, 0) x_{0}+G(t, 0) y_{0}+\int_{0}^{t} U(t, s) f(s) d s \quad \forall t \in I,
$$

where $U(t, s)=\int_{s}^{t} G(t, \tau) K(\tau, s) d \tau$.

Hypothesis H1. i) There exists an evolution operator $\{G(t, s)\}_{t, s \in I}$ associated to the family $\{A(t)\}_{t \geq 0}$.

ii) There exist $M, M_{0} \geq 0$ such that $|G(t, s)|_{B(X)} \leq M,\left|\frac{\partial}{\partial s} G(t, s)\right| \leq M_{0}$, for all $(t, s) \in \Delta$.

iii) $K(.,):. \Delta \rightarrow \mathbb{R}$ is continuous.

Hypothesis H2. i) $A$ is the infinitesimal generator of a strongly continuous and compact semigroup $\{\mathcal{G}(t) ; t \geq 0\}$ in $X$.

ii) There exists an operator $C: X \rightarrow X$ defined by

$$
C=\left[I+\sum_{i=1}^{m} a_{i} \mathcal{G}\left(t_{i}\right)\right]^{-1} .
$$

Let $m_{0} \geq 0$ be such that $|\mathcal{G}(t)| \leq m_{0} \forall t \in I$.

According to [4] if we assume that $\sum_{i=1}^{m}\left|a_{i}\right|<\frac{1}{m_{0}}$ then there exists $C$ as in Hypothesis $\mathrm{H} 2$ ii).

Definition 2.3. A continuous mapping $x(.) \in C(I, X)$ is called a mild solution of problem (1.3)-(1.4) if there exists a (Bochner) integrable function $f(.) \in L^{1}(I, X)$ such that

$$
\begin{gathered}
f(t) \in F(t, x(t)) \quad \text { a.e. }(I) \\
x(t)=\mathcal{G}(t) C x_{0}-\sum_{i=1}^{m} a_{i} \mathcal{G}(t) C \int_{0}^{t_{i}} \mathcal{G}\left(t_{i}-u\right) f(u) d u+\int_{0}^{t} \mathcal{G}(t-u) f(u) d u, t \in I .
\end{gathered}
$$

Remark 2.4. If we denote

$$
H(t, s)=\sum_{i=1}^{m} a_{i} \mathcal{G}(t) C \mathcal{G}\left(t_{i}-s\right) \chi_{\left[0, t_{i}\right]}(s)+\mathcal{G}(t-s) \chi_{[0, t]}(s),
$$

where $\chi_{S}(\cdot)$ is the characteristic function of the set $S$, then the solution $x(\cdot)$ in Definition 2.3 may be written as

$$
x(t)=\mathcal{G}(t) C x_{0}-\int_{0}^{T} H(t, s) f(s) d s .
$$

Obviously,

$$
|H(t, s)| \leq \sum_{i=1}^{m}\left|a_{i}\right| m_{0}^{2} \| C||+m_{0}=: m \quad \forall t, s \in I .
$$


In what follows $X$ is a real Banach space and we assume the following hypotheses. Hypothesis H3. i) $F(.,):. I \times X \rightarrow \mathcal{P}(X)$ has nonempty closed bounded values and for any $x \in X F(., x)$ is Lusin measurable on $I$.

ii) There exists $l(.) \in L^{1}(I,(0, \infty))$ such that, $\forall t \in I$

$$
\mathrm{d}_{H}\left(F\left(t, x_{1}\right), F\left(t, x_{2}\right)\right) \leq l(t)\left|x_{1}-x_{2}\right|, \quad \forall x_{1}, x_{2} \in X .
$$

iii) There exists $q(.) \in L^{1}(I,(0, \infty))$ such that $\forall t \in I$ we have

$$
F(t, 0) \subset q(t) B \text {. }
$$

Denote $L=\int_{0}^{T} l(s) d s$.

The technical results summarized in the following lemma are essential in the proof of our results. For the proof, we refer the reader to [15].

Lemma 2.5. i) Let $F_{i}: I \rightarrow \mathcal{P}(X), i=1$,2 be two Lusin measurable multifunctions and let $\varepsilon_{i}>0, i=1,2$ be such that

$$
H_{1}(t):=\left(F_{1}(t)+\varepsilon_{1} B\right) \cap\left(F_{2}(t)+\varepsilon_{2} B\right) \neq \emptyset, \quad \forall t \in I .
$$

Then the multifunction $H_{1}: I \rightarrow \mathcal{P}(X)$ has a Lusin measurable selection $h: I \rightarrow X$.

ii) Assume that Hypothesis $H 3$ is satisfied. Then for any continuous $x():. I \rightarrow$ $X, u():. I \rightarrow X$ measurable and any $\varepsilon>0$ one has

a) the multifunction $t \rightarrow F(t, x(t))$ is Lusin measurable on $I$.

b) the multifunction $H_{2}: I \rightarrow \mathcal{P}(X)$ defined by

$$
H_{2}(t):=(F(t, x(t))+\varepsilon B) \cap B_{X}(u(t), d(u(t), F(t, x(t)))+\varepsilon)
$$

has a Lusin measurable selection $g: I \rightarrow X$.

\section{The results}

Set $n(t)=\int_{0}^{t} l(u) \mathrm{d} u, t \in I$, denote $K_{0}:=\sup _{(t, s) \in \Delta}|K(t, s)|$ and note that

$$
|U(t, s)| \leq M K_{0}(t-s) \leq M K_{0} T \text {. }
$$

Theorem 3.1. We assume that Hypotheses H1 and H3 are satisfied. Then, for every $x_{0}, y_{0} \in X$, Cauchy problem (1.1) has a mild solution $x(.) \in C(I, X)$.

Proof. Let us first note that if $z():. I \rightarrow X$ is continuous, then every Lusin measurable selection $u: I \rightarrow X$ of the multifunction $t \rightarrow F(t, z(t))+B$ is Bochner integrable on $I$. More precisely, for any $t \in I$, there holds

$$
\begin{aligned}
|u(t)| & \leq d_{H}(F(t, z(t))+B, 0) \leq d_{H}(F(t, z(t)), F(t, 0))+d_{H}(F(t, 0), 0)+1 \\
& \leq l(t)|z(t)|+q(t)+1 .
\end{aligned}
$$

Let $0<\varepsilon<1, \quad \varepsilon_{n}=\frac{\varepsilon}{2^{n+2}}$.

Consider $f_{0}():. I \rightarrow X$, an arbitrary Lusin measurable, Bochner integrable function, and define

$$
x_{0}(t)=-\frac{\partial}{\partial s} G(t, 0) x_{0}+G(t, 0) y_{0}+\int_{0}^{t} U(t, s) f_{0}(s) d s, \quad t \in I .
$$


Since $x_{0}($.$) is continuous, by Lemma 2.5$ ii) there exists a Lusin measurable function $f_{1}():. I \rightarrow X$ which, for $t \in I$, satisfies

$$
f_{1}(t) \in\left(F\left(t, x_{0}(t)\right)+\varepsilon_{1} B\right) \cap B\left(f_{0}(t), d\left(f_{0}(t), F\left(t, x_{0}(t)\right)\right)+\varepsilon_{1}\right)
$$

Obviously, $f_{1}($.$) is Bochner integrable on I$. Define $x_{1}():. I \rightarrow X$ by

$$
x_{1}(t)=-\frac{\partial}{\partial s} G(t, 0) x_{0}+G(t, 0) y_{0}+\int_{0}^{t} U(t, s) f_{1}(s) d s, \quad t \in I .
$$

By induction, we construct a sequence $x_{n}: I \rightarrow X, n \geq 2$ given by

$$
x_{n}(t)=-\frac{\partial}{\partial s} G(t, 0) x_{0}+G(t, 0) y_{0}+\int_{0}^{t} U(t, s) f_{n}(s) d s, \quad t \in I,
$$

where $f_{n}():. I \rightarrow X$ is a Lusin measurable function which, for $t \in I$, satisfies:

$$
f_{n}(t) \in\left(F\left(t, x_{n-1}(t)\right)+\varepsilon_{n} B\right) \cap B\left(f_{n-1}(t), d\left(f_{n-1}(t), F\left(t, x_{n-1}(t)\right)\right)+\varepsilon_{n}\right) .
$$

At the same time, as we saw at the beginning of the proof, $f_{n}($.$) is also Bochner$ integrable.

From (3.2), for $n \geq 2$ and $t \in I$, we obtain

$$
\begin{aligned}
\left|f_{n}(t)-f_{n-1}(t)\right| & \leq d\left(f_{n-1}(t), F\left(t, x_{n-1}(t)\right)\right)+\varepsilon_{n} \\
& \leq d\left(f_{n-1}(t), F\left(t, x_{n-2}(t)\right)\right)+d_{H}\left(F\left(t, x_{n-2}(t)\right), F\left(t, x_{n-1}(t)\right)\right)+\varepsilon_{n} \\
& \leq \varepsilon_{n-1}+l(t)\left|x_{n-1}(t)-x_{n-2}(t)\right|+\varepsilon_{n} .
\end{aligned}
$$

Since $\varepsilon_{n-1}+\varepsilon_{n}<\varepsilon_{n-2}$, for $n \geq 2$, we deduce that

$$
\left|f_{n}(t)-f_{n-1}(t)\right| \leq \varepsilon_{n-2}+l(t)\left|x_{n-1}(t)-x_{n-2}(t)\right| .
$$

Denote $p_{0}(t):=d\left(f_{0}(t), F\left(t, x_{0}(t)\right)\right), t \in I$. We next prove by recurrence, that for $n \geq 2$ and $t \in I$

$$
\begin{aligned}
\left|x_{n}(t)-x_{n-1}(t)\right| & \leq \sum_{k=0}^{n-2} \int_{0}^{t} \varepsilon_{n-2-k} \frac{\left(M K_{0} T\right)^{k+1}(n(t)-n(u))^{k}}{k !} d u \\
& +\varepsilon_{0} \int_{0}^{t} \frac{\left(M K_{0} T\right)^{n}(n(t)-n(u))^{n-1}}{(n-1) !} d u \\
& +\int_{0}^{t} \frac{\left(M K_{0} T\right)^{n}(n(t)-n(u))^{n-1}}{(n-1) !} p_{0}(u) d u .
\end{aligned}
$$

We start with $n=2$. In view of (3.1), (3.2) and (3.3), for $t \in I$, there is

$$
\begin{gathered}
\left|x_{2}(t)-x_{1}(t)\right| \leq \int_{0}^{t}|U(t, s)| \cdot\left|f_{2}(s)-f_{1}(s)\right| d s \\
\leq \int_{0}^{t} M K_{0} T\left[\varepsilon_{0}+l(s)\left|x_{1}(s)-x_{0}(s)\right|\right] d s \\
\leq \varepsilon_{0} M K_{0} T t+\int_{0}^{t}\left[M K_{0} T l(s) \int_{0}^{s}|U(s, r)| \cdot\left|f_{1}(r)-f_{0}(r)\right| d r\right] d s \\
\leq \varepsilon_{0} M K_{0} T t+\int_{0}^{t}\left[\left(M K_{0} T\right)^{2} l(s) \int_{0}^{s}\left(p_{0}(u)+\varepsilon_{1}\right) d u\right] d s
\end{gathered}
$$




$$
\begin{aligned}
& \leq \varepsilon_{0} M K_{0} T t+\int_{0}^{t}\left[\left(M K_{0} T\right)^{2}\left(p_{0}(u)+\varepsilon_{1}\right) \int_{u}^{t} l(s) d s\right] d u \\
& =\varepsilon_{0} M K_{0} T t+\int_{0}^{t}\left(M K_{0} T\right)^{2}(n(t)-n(s))\left[p_{0}(s)+\varepsilon_{0}\right] d s,
\end{aligned}
$$

i.e, (3.4) is verified for $n=2$.

Using again (3.3) and (3.4), we conclude

$$
\begin{aligned}
& \left|x_{n+1}(t)-x_{n}(t)\right| \leq \int_{0}^{t}|U(t, s)| \cdot\left|f_{n+1}(s)-f_{n}(s)\right| d s \\
& \leq \int_{0}^{t} M K_{0} T\left[\varepsilon_{n-1}+l(s)\left|x_{n}(s)-x_{n-1}(s)\right|\right] d s \\
& \leq \varepsilon_{n-1} M K_{0} T t+\int_{0}^{t} l(s)\left[\sum_{k=0}^{n-2} \int_{0}^{s} \varepsilon_{n-2-k} \frac{\left(M K_{0} T\right)^{k+2}(n(s)-n(u))^{k}}{k !} d u\right. \\
& \left.+\int_{0}^{s} \frac{\left(M K_{0} T\right)^{n+1}(n(s)-n(u))^{n-1}}{(n-1) !}\left(p_{0}(u)+\varepsilon_{0}\right) d u\right] d s \\
& =\varepsilon_{n-1} M K_{0} T t+\sum_{k=0}^{n-2} \varepsilon_{n-2-k} \int_{0}^{t}\left[\int_{0}^{s} \frac{\left(M K_{0} T\right)^{k+2}(n(s)-n(u))^{k}}{k !} l(s) d u\right] d s \\
& +\int_{0}^{t} l(s)\left(\int_{0}^{s} \frac{\left(M K_{0} T\right)^{n+1}(n(s)-n(u))^{n-1}}{(n-1) !} l(s)\left[p_{0}(u)+\varepsilon_{0}\right] d u\right) d s \\
& =\varepsilon_{n-1} M K_{0} T t+\sum_{k=0}^{n-2} \varepsilon_{n-2-k} \int_{0}^{t}\left(\int_{u}^{t} \frac{\left(M K_{0} T\right)^{k+2}(n(s)-n(u))^{k}}{k !} l(s) d s\right) d u \\
& +\int_{0}^{t}\left(\int_{u}^{t} \frac{\left(M K_{0} T\right)^{n+1}(n(s)-n(u))^{n-1}}{(n-1) !} l(s) d s\right)\left[p_{0}(u)+\varepsilon_{0}\right] d u \\
& =\varepsilon_{n-1} M K_{0} T t+\sum_{k=0}^{n-2} \varepsilon_{n-2-k} \int_{0}^{t} \frac{\left(M K_{0} T\right)^{k+2}(n(s)-n(u))^{k+1}}{(k+1) !} d u \\
& +\int_{0}^{t} \frac{\left(M K_{0} T\right)^{n+1}(n(s)-n(u))^{n}}{n !}\left[p_{0}(u)+\varepsilon_{0}\right] d u \\
& =\sum_{k=0}^{n-1} \varepsilon_{n-1-k} \cdot \int_{0}^{t} \frac{\left(M K_{0} T\right)^{k+1}(n(s)-n(u))^{k}}{k !} d u \\
& +\int_{0}^{t} \frac{\left(M K_{0} T\right)^{n+1}(n(s)-n(u))^{n}}{n !}\left[p_{0}(u)+\varepsilon_{0}\right] d u
\end{aligned}
$$

and statement (3.8) it is true for $n+1$.

From (3.8) it follows that for $n \geq 2$ and $t \in I$

$$
\left|x_{n}(t)-x_{n-1}(t)\right| \leq a_{n},
$$

where

$$
a_{n}=\sum_{k=0}^{n-2} \varepsilon_{n-2-k} \frac{\left(M K_{0} T\right)^{k+1} n(T)^{k}}{k !}+\frac{\left(M K_{0} T\right)^{n} n(T)^{n-1}}{(n-1) !}\left[\int_{0}^{1} p_{0}(u) d u+\varepsilon_{0}\right],
$$


Obviously, the series whose $n$-th term is $a_{n}$ converges. So, from (3.5) we infer that $x_{n}($.$) converges to a continuous function, x():. I \rightarrow X$, uniformly on $I$.

On the other hand, in view of (3.3) there is

$$
\left|f_{n}(t)-f_{n-1}(t)\right| \leq \varepsilon_{n-2}+l(t) a_{n-1}, \quad t \in I, n \geq 3
$$

which implies that the sequence $f_{n}($.$) converges to a Lusin measurable function$ $f(\cdot): I \rightarrow X$.

Since $x_{n}($.$) is bounded and$

$$
\left|f_{n}(t)\right| \leq l(t)\left|x_{n-1}(t)\right|+q(t)+1,
$$

we infer that $f($.$) is also Bochner integrable.$

Passing with $n \rightarrow \infty$ in (3.1) and using the Lebesgue dominated convergence theorem, we obtain

$$
x(t)=-\frac{\partial}{\partial s} G(t, 0) x_{0}+G(t, 0) y_{0}+\int_{0}^{t} U(t, s) f(s) d s, \quad t \in I .
$$

On the other hand, from (3.2) we get

$$
f_{n}(t) \in F\left(t, x_{n}(t)\right)+\varepsilon_{n} B, \quad t \in I, n \geq 1
$$

and letting $n \rightarrow \infty$ we obtain

$$
f(t) \in F(t, x(t)), \quad t \in I,
$$

which completes the proof.

Theorem 3.2. Assume that Hypotheses H2 and H3 are satisfied and $m L<1$.

Then, for every $x_{0} \in X$ problem (1.3)-(1.4) has a solution $x():. I \rightarrow X$.

Proof. The proof follows the same pattern as in the proof of Theorem 3.1. This time

$$
x_{n}(t)=\mathcal{G}(t) C x_{0}-\int_{0}^{T} H(t, s) f_{n}(s) \mathrm{d} s, \quad \forall t \in I,
$$

with $f_{n}(\cdot)$ as before and

$$
\left|x_{n}(t)-x_{n-1}(t)\right| \leq \sum_{j=0}^{n-2} \varepsilon_{n-2-j} m^{j+1} L^{j} T+m^{n} L^{n-1} \int_{0}^{T}\left(p_{0}(s)+\varepsilon_{0}\right) d s
$$

for $n \geq 2$ and $t \in I$. The estimate in (3.5) becames

$$
\left|x_{n}(t)-x_{n-1}(t)\right| \leq a_{n},
$$

where

$$
a_{n}=\sum_{j=0}^{n-2} \varepsilon_{n-2-j} m^{j+1} L^{j} T+m^{n} L^{n-1} \int_{0}^{T}\left(p_{0}(s)+\varepsilon_{0}\right) d s
$$

Taking into account the fact that $m L<1$, we deduce that the series whose $n$-th term is $a_{n}$ is convergent. 


\section{References}

[1] Aubin, J.P., Frankowska, H., Set-valued Analysis, Birkhauser, 1990.

[2] Baliki, A., Benchohra, M., Graef, J.R., Global existence and stability of second order functional evolution equations with infinite delay, Electronic J. Qual. Theory Differ. Equations, 2016(2016), no. 23, 1-10.

[3] Baliki, A., Benchohra, M., Nieto, J.J., Qualitative analysis of second-order functional evolution equations, Dynamic Syst. Appl., 24(2015), 559-572.

[4] Benchohra, M., Gatsori, E.P., Ntouyas, S.K., Controllability results for semilinear evolution inclusions with nonlocal conditions, J. Optim. Theory Appl., 118(2003), 493-513.

[5] Benchohra, M., Medjadj, I., Global existence results for second order neutral functional differential equations with state-dependent delay, Comment. Math. Univ. Carolin., 57(2016), 169-183.

[6] Benchohra, M., Ntouyas, S.K., Existence of mild solutions of semilinear evolution inclusions with nonlocal conditions, Georgian Math. J., 7(2000), 221-230.

[7] Bressan, A., Colombo, G., Extensions and selections of maps with decomposable values, Studia Math., 90(1988), 69-86.

[8] Byszewski, L., Application of properties of the right-hand sides of evolution equations to an investigation of nonlocal evolution problems, Nonlin. Anal., 33(1998), 413-426.

[9] Byszewski, L., Existence and uniqueness of a classical solution to a functional-differential abstract nonlocal Cauchy problem, J. Appl. Math. Stochastic Anal., 12(1999), 91-97.

[10] Cernea, A., On an evolution inclusion in non separable Banach spaces, Opuscula Math., 29(2009), 131-138.

[11] Cernea, A., A note on a semilinear evolution inclusion with nonlocal conditions, ROMAI J., 12(2016), 33-38.

[12] Cernea, A., A note on the solutions of a second-order evolution inclusion in non separable Banach spaces, Comment. Math. Univ. Carolin., 58(2017), 307-314.

[13] Cernea, A., Some remarks on the solutions of a second-order evolution inclusion, Dynamic Syst. Appl., 27(2018), 319-330.

[14] Cernea, A., On the mild solutions of a class of second-order integro-differential inclusions, J. Nonlinear Variational Anal., 3(2020), 247-256.

[15] De Blasi F.S., Pianigiani, G., Evolution inclusions in non separable Banach spaces, Comment. Math. Univ. Carolin., 40(1999), 227-250.

[16] Filippov, A.F., Classical solutions of differential equations with multivalued right-hand side, SIAM J. Control Optim., 5(1967), 609-621.

[17] Henriquez, H.R., Existence of solutions of nonautonomous second order functional differential equations with infinite delay, Nonlinear Anal., 74(2011), 3333-3352.

[18] Henriquez, H.R., Poblete, V., Pozo, J.C., Mild solutions of non-autonomous second order problems with nonlocal initial conditions, J. Math. Anal. Appl., 412(2014), 1064-1083.

[19] Kozak, M., A fundamental solution of a second-order differential equation in a Banach space, Univ. Iagel. Acta. Math., 32(1995), 275-289.

[20] Kuratowski, K., Ryll-Nardzewski, C., A general theorem on selectors, Bull. Acad. Pol. Sci. Math. Astron. Phys., 13(1965), 397-403. 
Aurelian Cernea

University of Bucharest,

Faculty of Mathematics and Computer Sciences

14, Academiei Street, 010014 Bucharest,

Academy of Romanian Scientists,

Splaiul Independenței 54,

050094 Bucharest, Romania

e-mail: acernea@fmi.unibuc.ro 